\title{
Compact Electrospray Ionization Ion Mobility Spectrometer
}

\author{
Christian Thoben, Christian-Robert Raddatz, André Ahrens, Janna Birte Schulte, Arend Staschel and \\ Stefan Zimmermann

\begin{abstract}
Institute of Electrical Engineering and Measurement Technology, Leibniz University Hannover, Appelstr. 9A, 30167 Hannover, Germany

Contact: thoben@geml.uni-hannover.de
\end{abstract}

\section{Introduction}

Ion mobility spectrometers (IMS) are typically used for fast and sensitive detection of substances in the gas phase. IMS offer detection limits in the low ppt range with measurement times of less than one second. The measurement principle is based on the separation of different ion species according to their ion-specific mobility in a neutral drift gas under the influence of an electric field. [1]

Nowadays, IMS are well established in different analytical applications, e.g., in safety and security such as the detection of chemical warfare agents $[2,3]$, explosives [4,5] or drugs of abuse [6]. Especially in food quality monitoring and food analysis ion mobility spectrometry is becoming increasingly important $[7-10]$. Furthermore, a variety of other applications exist, e.g. breath analysis [11], further medical applications $[12,13]$ and environmental analysis [14].

For some of these applications, low-cost and mobile instruments are required. Especially in the field of water pollution control, there is a lack of compact devices that can rapidly detect pollutants after accidental or deliberate release and assess the current situation. The aim of this work is to close this gap with preliminary steps towards the development of a portable instrument for on-site process control and detection of contaminants in water. For this purpose, three different compact and low-cost IMS are compared with our standard PEEK-IMS system in terms of their suitability for the analysis of liquid samples.

Here, electrospray ionization (ESI) is used to ionize and transfer liquid samples into the gas phase. An electrospray is induced from a liquid sample in a capillary under the influence of a strong electric field, so that a dispersed nebula of charged droplets is formed. Finally, ions are generated in the gas phase by a complex process involving evaporation of the solvent and droplet jets fissions due to coulombic stresses [15].

\section{Experimental}

The IMS investigated in this work mainly consist of a drift region and a desolvation region. In the drift region, the ions are separated and detected according to their mobility. In the desolvation region, ions are formed from charged droplets previously gener- ated by an electrospray. The two regions are separated by a tristate ion shutter, which injects the ions into the drift region [16].

The global parameters applied to all experiments are listed in Tab. 1. The individual systems in this work differ mainly in the dimensions and the electric field strength used in the desolvation and drift region. The corresponding parameters are listed in Tab. 2-5 for the respective systems. A detailed description of our standard PEEK-IMS can be found elsewhere [16]. Furthermore, the setup of the miniaturized, low-cost PEEK-IMS system is described in [17].

Another possibility for compact IMS at low cost is the use of printed circuit boards [18-20]. With this manufacturing technique it is also possible to simply rescale an IMS design. A detailed description of the miniaturized, low-cost PCB-ESI-IMS presented in this work can be found in [21]. A size comparison of all systems used in this work can be seen in Fig. 1.

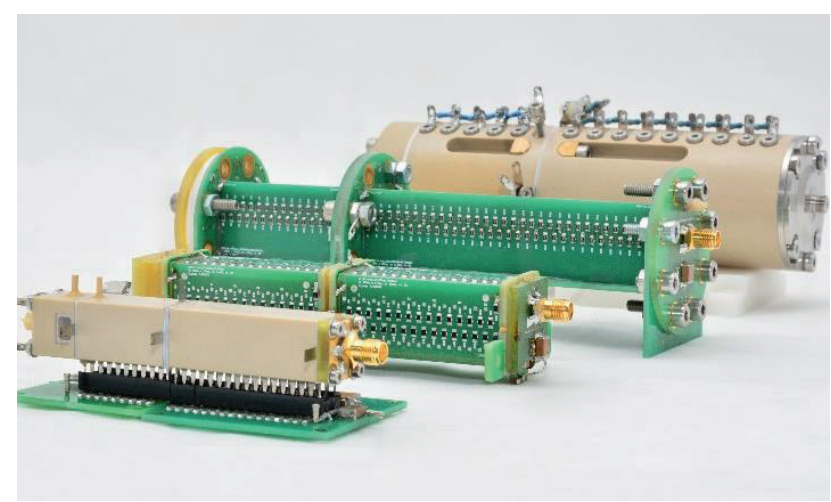

Fig. 1: Photo of the different ESI-IMS. From front to back: miniaturized PEEK-IMS, miniaturized, low-cost PCB-IMS, low-cost PCB-IMS and standard PEEK-IMS.

To supply sample fluids to the electrospray ionization source, we use an Advanced Microfluidics LSPone programmable syringe pump with a $50-\mu l$ syringe and New Objective Metal Taper Tip emitters with an inner diameter of $50 \mu \mathrm{m}$. The flow rate is set to $1 \mu \mathrm{l} / \mathrm{min}$. The ESI voltage is applied between the emitter and the grounded first ring of the desolvation region. In addition, the IMS is supplied with a drift gas flow of $250 \mathrm{ml} / \mathrm{min}$ resp. $120 \mathrm{ml} / \mathrm{min}$ of purified dry air flowing in the opposite direction to the spray direction. 
Tab. 1: Global operating parameters

\begin{tabular}{|l|l|}
\hline Global parameters & Value \\
\hline Emitter voltage & $2-3 \mathrm{kV}$ \\
\hline Emitter diameter & $50 \mu \mathrm{m}$ \\
\hline Drift gas & Purified dry air \\
\hline Drift gas dew point & $-85^{\circ} \mathrm{C}$ \\
\hline Pressure & $1013 \mathrm{mbar}$ \\
\hline Temperature & $23^{\circ} \mathrm{C}$ \\
\hline
\end{tabular}

Tab. 2: Operating parameters standard PEEK-IMS

\begin{tabular}{|l|l|}
\hline Parameter & Value \\
\hline Length of drift region & $75 \mathrm{~mm}$ \\
\hline Length of desolvation region & $50 \mathrm{~mm}$ \\
\hline Field strength & $60 \mathrm{~V} / \mathrm{mm}$ \\
\hline Drift gas flow rate & $250 \mathrm{ml} / \mathrm{min}$ \\
\hline Outer diameter & $48 \mathrm{~mm}$ \\
\hline
\end{tabular}

Tab. 3: Operating parameters low-cost PCB-IMS

\begin{tabular}{|l|l|}
\hline Parameter & Value \\
\hline Length of drift region & $100 \mathrm{~mm}$ \\
\hline Length of desolvation region & $50 \mathrm{~mm}$ \\
\hline Field strength & $73 \mathrm{~V} / \mathrm{mm}$ \\
\hline Drift gas flow rate & $250 \mathrm{ml} / \mathrm{min}$ \\
\hline Outer cross section & $28 \mathrm{~mm} \times 28 \mathrm{~mm}$ \\
\hline
\end{tabular}

Tab. 4: Operating parameters miniaturized PEEK-IMS

\begin{tabular}{|l|l|}
\hline Parameter & Value \\
\hline Length of drift region & $40 \mathrm{~mm}$ \\
\hline Length of desolvation region & $25 \mathrm{~mm}$ \\
\hline Field strength & $61 \mathrm{~V} / \mathrm{mm}$ \\
\hline Drift gas flow rate & $120 \mathrm{ml} / \mathrm{min}$ \\
\hline Outer cross section & $15 \mathrm{~mm} \mathrm{x} 15 \mathrm{~mm}$ \\
\hline
\end{tabular}

Tab. 5: Operating parameters miniaturized, low-cost PCB-IMS

\begin{tabular}{|l|l|}
\hline Parameter & Value \\
\hline Length of drift region & $50 \mathrm{~mm}$ \\
\hline Length of desolvation region & $50 \mathrm{~mm}$ \\
\hline Field strength & $50 \mathrm{~V} / \mathrm{mm}-$ \\
& $100 \mathrm{~V} / \mathrm{mm}$ \\
\hline Drift gas flow rate & $120 \mathrm{ml} / \mathrm{min}$ \\
\hline Outer cross section & $25 \mathrm{~mm} \times 25 \mathrm{~mm}$ \\
\hline
\end{tabular}

\section{Ion Mobility Spectrometry}

The ion mobility $K$ can be measured experimentally, namely by the drift time $t_{D}$ required for an ion to traverse the drift tube of length $L$ in the presence of the electric drift field $E$.

$$
K=\frac{L}{t_{D} \cdot E}
$$

Often, the ion mobility $K$ from in Eq. (1) is converted to a reduced mobility value $K_{0}$ by normalizing the expression to temperature and pressure.

$$
K_{0}=K \cdot \frac{p}{1013 \mathrm{mbar}} \cdot \frac{273 \mathrm{~K}}{T}
$$

where $p$ is the operating pressure in mbar and $T$ is the operating temperature in Kelvin. Hereby, a better comparison between the different systems is possible. However, this normalization does not account for variations in ion chemistry due to different conditions in the ionization region.

As described in [22], the signal-to-noise ratio improves with drift voltages higher than the optimum drift voltage for maximum resolving power, while the resolving power just slightly degrades. This effect is stronger in shorter drift tubes, since lower optimum drift voltages are required.

\section{Electrospray Ionization}

First, the flow rate of the analytic solution affects the ionization efficiency of undissociated substances in ESI-IMS [23,24]. Secondly, in addition to the analytes themselves, the properties of the solvent are the main determinants of the ionization efficiency in ESI.

The Hendricks equation (3) provides an approximation to describe the electrospray ion current $i_{E S}$, which is related to the solvent flow rate $v_{f}$ and the solution conductivity $\sigma$, as well as the electric field strength at the emitter $E$.

$$
i_{E S}=A_{H} \cdot v_{f}^{0.57} \cdot E^{0.43} \cdot \sigma^{0.43}
$$

where $A_{H}$ is an evaluable constant that depends on the dielectric constant and surface tension of the solvent. Increasing the solvent flow rate or the solution conductivity results in a higher electrospray ion current [25].

\section{Results and Discussion}

In a first experimental setup, we compare our standard PEEK-IMS with a low-cost PCB-IMS, a miniaturized PEEK-IMS, and a miniaturized, lowcost PCB-IMS. As shown in Fig. 2, similar results are obtained for a pure 80:20 methanol:water solvent mixture. Accordingly, all systems are suitable for electrospray ionization. However, with a length of $150 \mathrm{~mm}$, the PCB-IMS is relatively long, so high absolute voltages must be provided. Furthermore, with a relatively high given voltage supply of $12.5 \mathrm{kV}$, a further increase in drift voltage is not possible to improve the signal-to-noise ratio. Therefore, the IMS drift tube should be miniaturized first and foremost. 


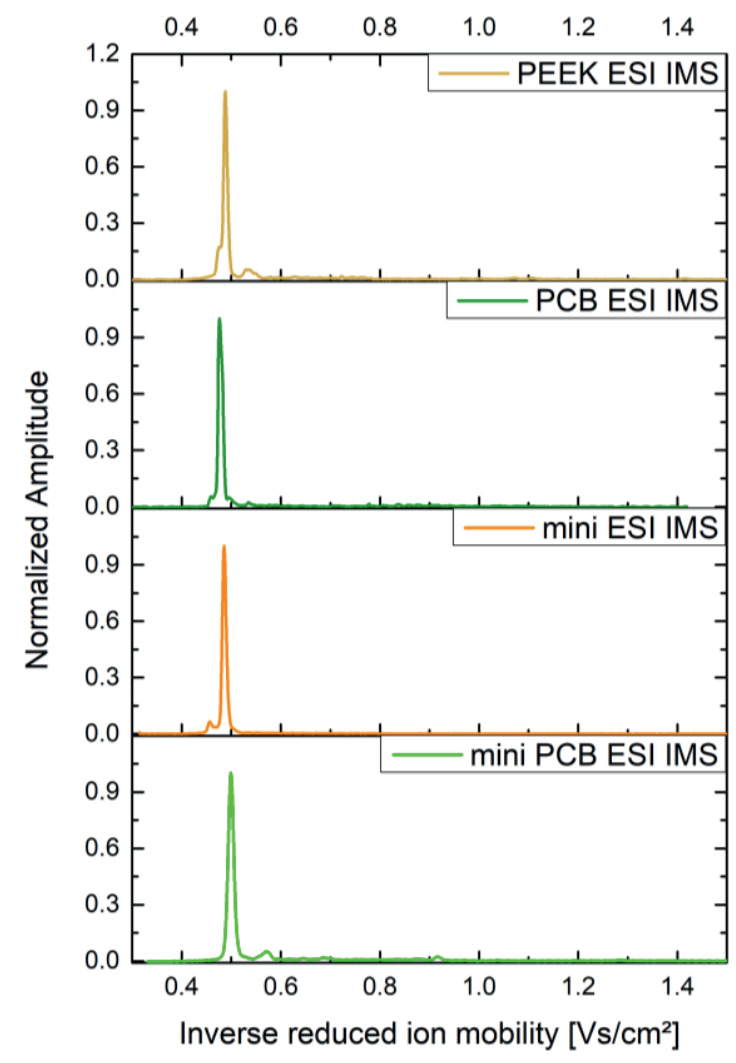

Fig. 2: Spectra of a pure 80:20 methanol:water mixture measured with the standard PEEK-IMS (ocher), the lowcost PCB-IMS (dark green), the miniaturized PEEK-IMS (orange) and the miniaturized, low-cost PCB-IMS (light green).

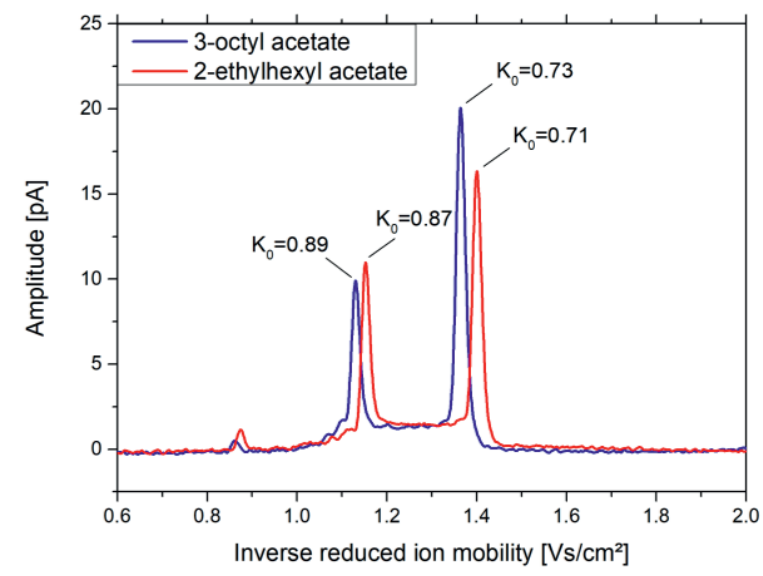

Fig. 3: Spectra of the isomers $0.1 \mathrm{~mol} / 13$-octyl acetate and $0.1 \mathrm{~mol} / 1$ 2-ethylhexyl acetate in 70:30 ethanol:water with $5 \mathrm{mmol} / \mathrm{l}$ sodium acetate measured with the miniaturized PEEK-ESI-IMS.

A notable advantage of IMS is the separation of isomers. As an example, the spectra of two aroma compounds, the flavoring 3-octyl acetate and 2ethylhexyl acetate used as solvent and additive in detergents, with the same mass are shown in Fig. 3.
As it can be seen in Fig. 3, each analyte forms three peaks, whereby the individual dimers and trimers have different $K_{0}$ values for the two isomers, enabling both substances to be differentiated. Hereby, the miniaturized PEEK-ESI-IMS was used and to demonstrate the potential for analyzing liquid samples even with very compact and low-cost IMS. However, there is no baseline separation of the two analytes.

Another approach for manufacturing small and lowcost IMS is the use of PCBs. One advantage of short drift tubes is that the drift voltage can easily be increased above the optimum for best resolving power, giving a better signal-to-noise ratio. For demonstration, the herbicide isoproturon was measured with the miniaturized, low-cost PCB-IMS at increased drift voltages. As shown in Fig. 4, the amplitude of the isoproturon peak increases significantly as the drift voltage is increased.

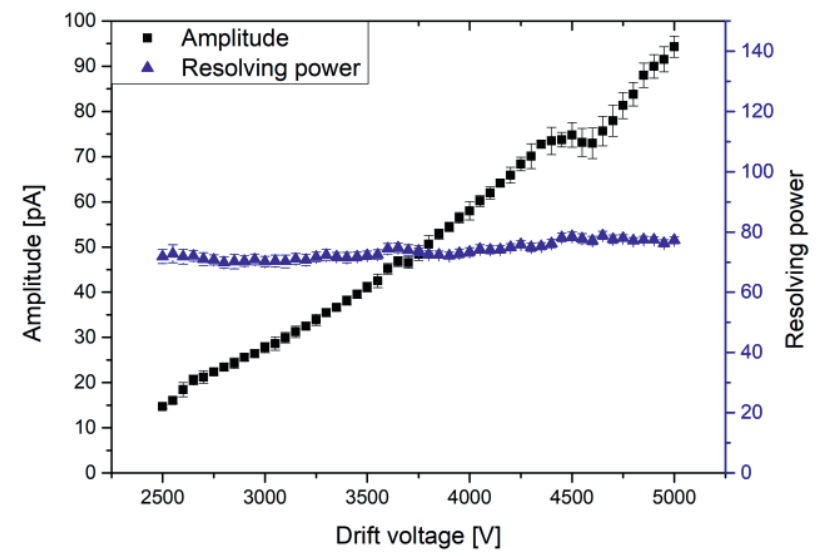

Fig. 4: Peak Amplitude of $10 \mathrm{mg} / \mathrm{l}$ isoproturon in 80:20 methanol:water measured with the miniaturized, low-cost PCB-IMS plotted versus the drift voltage.

In contrast to the theory, the resolving power remains relatively constant. With the drift voltage, we simultaneously increase the desolvation voltage. As shown in [16], by increasing the electric field strength in the desolvation region, we also influence the voltage at the ESI emitter. This in turn influences the formation of ions from the electrospray, so that apparently other clusters are formed here.

\section{Conclusion}

As presented in this work, different IMS designs are suitable for use with electrospray ionization. Consequently, several factors are applicable as evaluation criteria depending on the application, such as the size, the temperature resistance of the material, and the cost. Compact IMS drift tubes have the advantage of requiring lower drift voltage supplies also shrinking the periphery. Furthermore, drift voltages above the optimum drift voltage regarding the maxi- 
mum resolving power are easily possible to increase the signal-to-noise ratio.

\section{References}

[1] G.A. Eiceman, Z. Karpas, H.H. Hill, Ion mobility spectrometry, 3rd ed., CRC Press, Boca Raton, 2013.

[2] M.A. Mäkinen, O.A. Anttalainen, Sillanpää, Mika E $\mathrm{T}$, lon mobility spectrometry and its applications in detection of chemical warfare agents, Anal. Chem. 82 (2010) 9594-9600.

[3] J. Puton, J. Namieśnik, Ion mobility spectrometry: Current status and application for chemical warfare agents detection, TrAC, Trends Anal. Chem. 85 (2016) 10-20.

[4] M. Tabrizchi, V. Ilbeigi, Detection of explosives by positive corona discharge ion mobility spectrometry, Journal of hazardous materials 176 (2010) 692-696.

[5] H. Shahraki, M. Tabrizchi, H. Farrokhpor, Detection of explosives using negative ion mobility spectrometry in air based on dopant-assisted thermal ionization, Journal of hazardous materials 357 (2018) 19.

[6] A.B. Kanu, H.H. Hill, Identity confirmation of drugs and explosives in ion mobility spectrometry using a secondary drift gas, Talanta 73 (2007) 692-699.

[7] M. Hernández-Mesa, A. Escourrou, F. Monteau, B. Le Bizec, G. Dervilly-Pinel, Current applications and perspectives of ion mobility spectrometry to answer chemical food safety issues, TrAC Trends in Analytical Chemistry 94 (2017) 39-53.

[8] M.-Y. Piñero, M. Amo-González, R.D. Ballesteros, L.R. Pérez, G.F. de La Mora, L. Arce, Chemical Fingerprinting of Olive Oils by Electrospray Ionization-Differential Mobility Analysis-Mass Spectrometry: A New Alternative to Food Authenticity Testing, J. Am. Soc. Mass Spectrom. 31 (2020) 527-537.

[9] M.J. Aliaño-González, M. Ferreiro-González, E. Espada-Bellido, M. Palma, G.F. Barbero, A Screening Method Based on Headspace-Ion Mobility Spectrometry to Identify Adulterated Honey, Sensors 19 (2019).

[10] N. Gerhardt, M. Birkenmeier, D. Sanders, S. Rohn, P. Weller, Resolution-optimized headspace gas chromatography-ion mobility spectrometry (HS-GCIMS) for non-targeted olive oil profiling, Anal Bioanal Chem 409 (2017) 3933-3942.

[11] M. Malásková, D. Olivenza-León, P.D. Chellayah, J. Martini, W. Lederer, V. Ruzsanyi, K. Unterkofler, P. Mochalski, T.D. Märk, P. Watts, C.A. Mayhew, Studies pertaining to the monitoring of volatile halogenated anaesthetics in breath by proton transfer reaction mass spectrometry, Journal of breath research 14 (2020) 26004.

[12] I.D. Campuzano, J.L. Lippens, Ion mobility in the pharmaceutical industry: An established biophysical technique or still niche?, Current Opinion in Chemical Biology 42 (2018) 147-159.

[13] C. Thoben, N. Dennhardt, T. Krauß, R. Sümpelmann, S. Zimmermann, H. Rüffert, S. Heiderich, Preparation of anaesthesia workstation for triggerfree anaesthesia: An observational laboratory study, European journal of anaesthesiology 36 (2019) 851-856.
[14] I. Márquez-Sillero, E. Aguilera-Herrador, S. Cárdenas, M. Valcárcel, lon-mobility spectrometry for environmental analysis, TrAC, Trends Anal. Chem. 30 (2011) 677-690.

[15] P. Kebarle, U.H. Verkerk, Electrospray: from ions in solution to ions in the gas phase, what we know now, Mass Spectrom Rev 28 (2009) 898-917.

[16] C. Thoben, C.-R. Raddatz, M. Lippmann, Z. Salehimoghaddam, S. Zimmermann, Electrospray ionization ion mobility spectrometer with new tristate ion gating for improved sensitivity for compounds with lower ion mobility, Talanta 233 (2021) 122579.

[17] A. Ahrens, M. Hitzemann, S. Zimmermann, Miniaturized high-performance drift tube ion mobility spectrometer, Int. J. Ion Mobil. Spec. 22 (2019) 7783.

[18] T. Reinecke, B.H. Clowers, Implementation of a flexible, open-source platform for ion mobility spectrometry, HardwareX 4 (2018) e00030.

[19] N. Chantipmanee, P.C. Hauser, Development of simple drift tube design for ion mobility spectrometry based on flexible printed circuit board material, Analytica Chimica Acta 1170 (2021) 338626.

[20] A. Bohnhorst, A.T. Kirk, S. Zimmermann, Simulation aided design of a low cost ion mobility spectrometer based on printed circuit boards, Int. J. Ion Mobil. Spec. 19 (2016) 167-174.

[21] A. Bohnhorst, A.T. Kirk, S. Zimmermann, Toward Compact High-Performance Ion Mobility Spectrometers: Ion Gating in Ion Mobility Spectrometry, Anal. Chem. 93 (2021) 6062-6070.

[22] A.T. Kirk, S. Zimmermann, An analytical model for the optimum drift voltage of drift tube ion mobility spectrometers with respect to resolving power and detection limits, Int. J. Ion Mobil. Spec. 18 (2015) 129-135.

[23] M.T. Jafari, Improved design for high resolution electrospray ionization ion mobility spectrometry, Talanta 77 (2009) 1632-1639.

[24] X. Tang, J.E. Bruce, H.H. Hill, Characterizing electrospray ionization using atmospheric pressure ion mobility spectrometry, Anal. Chem. 78 (2006) 7751-7760.

[25] P. Kebarle, L. Tang, From ions in solution to ions in the gas phase - the mechanism of electrospray mass spectrometry, Anal. Chem. 65 (1993) 972A986A.

\section{Acknowledgements}

Supported by the German Federal Ministry for Economic Affairs and Energy (BMWi) on the basis of a decision by the German Bundestag, Grant 20666 N, and funded by the Deutsche Forschungsgemeinschaft (DFG, German Research Foundation) - 396430937. 